\title{
Impact of Signal Preprocessing on the Inverse Localization of the Origin of Ventricular Pacing
}

\author{
Jana Svehlikova ${ }^{1}$, Jan Zelinka ${ }^{1}$, Yesim Serinagaoglu Dogrusoz ${ }^{2}$, Wilson Good $^{3}$, Milan Tysler ${ }^{1}$, \\ Laura Bear ${ }^{4}$ \\ ${ }^{1}$ Institute of Measurement Science, Slovak Academy of Sciences, Bratislava, Slovakia \\ ${ }^{2}$ Electrical and Electronics Engineering Department, METU, Turkey \\ ${ }^{3}$ Scientific Computing and Imaging Institute, University of Utah, Salt Lake City, UT, USA \\ ${ }^{4}$ Electrophysiology and Heart Modelling Institute (IHU-LIRYC), Bordeaux, France
}

\begin{abstract}
The ECG signal measured in clinical or experimental conditions is usually contaminated by noise, which should be eliminated by proper signal processing methods. The selection of the method can be crucial for the results obtained from measured signal.

Seven methods of high frequency noise removal and four methods of baseline drift removal were applied on experimental ECG signals measured during $30 \mathrm{~s}$ of induced ventricular pacing. Then for all possible combinations of the signal pre-processing methods an averaged signal representing one heart beat was computed. The origin of ventricular activity was assessed by the inverse solution using a single dipole. The impact of pre-processing on the quality of the inverse solution was observed.

The localization error between the known position of the stimulating electrode and the computed origin of ventricular activation was more than $5 \mathrm{~cm}$ if the baseline of the processed signal did not coincide with the zero line. However, if the processed signal started from the zero value, an acceptable localization error from 0.7 tol. $3 \mathrm{~cm}$ was obtained.

The inverse assessment of the origin of ventricular tachycardia using a single dipole is very sensitive to proper baseline drift removal. Because the inverse solution was computed for averaged signal, various methods of high frequency noise removal did not affect the results.
\end{abstract}

\section{Introduction}

The study was performed within the Signal Preprocessing Group of the Consortium for ECG imaging CEI (www.ecg-imaging.org). One application of electrocardiographic imaging (ECGI) is the localization of the origin of ventricular tachycardia (VT) or premature ventricular contraction (PVC), which is the aim of various research groups [1], [2], [3]. Such undesired starting point of ventricular activity is often eliminated by the invasive radiofrequency ablation. Preliminary noninvasive localization of VT/PVC origin can shorten the consequent ablation procedure. This leads to the use of various inverse methods in electrocardiology. Some of them use complex models describing the ventricular activation in the whole volume such as transmembrane voltages, epicardial potentials or equivalent double layer [4], [5]. However, a starting point of ventricular electrical activity can be localized also using a model of a single point electrical generator as it was shown on simulated data in [6]. The inverse methods in electrocardiography solve the ill-posed problems, thus they can be sensitive to changes in the input data. In experimental or clinical conditions, many factors can influence either the quality of a torso model or the quality of measured signals.

The aim of the Signal Preprocessing Group of the CEI was to compare different signal preprocessing techniques applied on the ECG signal measured under the real conditions and study their impact on the results of the inverse solution. The presented study used the inverse localization of the origin of ventricular pacing using one dipole.

\section{Materials and Method}

The experimental ECG signals were obtained from a Langendorf-perfused pig heart placed in a human-shaped torso tank, provided by the Electrophysiology and Heart Modelling Institute (IHU-LIRYC), France. Simultaneous measurements of 108 epicardial signals on the sock (Figure 1) together with 128 signals on the tank (Figure 2) were performed during $30 \mathrm{~s}$ of right ventricular (RV) pacing. Several signal preprocessing methods were then applied on the original measured signal. 


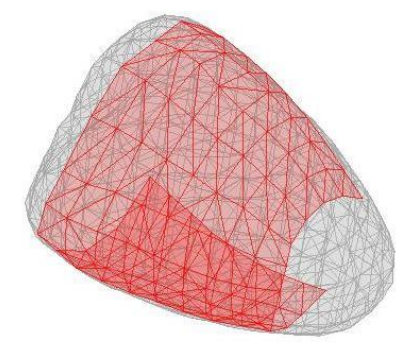

Figure 1. Triangulated epicardial surface (grey) and the sock (red) with 108 measuring electrodes.

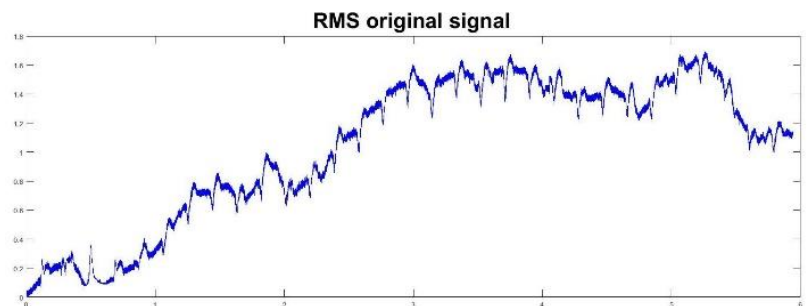

Figure 2. Root-mean-squared values of the original 128 signals measured on the tank in each time instant out of 30 seconds.

First, seven methods for high frequency noise removal (HFR 1-7) were used. HFR1 was a simple moving average filter computed over the $20 \mathrm{~ms}$ time window, HFR2 was so called Pipberger's filter suggested in [7] where the averaging window corresponds with the length of two $50 \mathrm{~Hz}$ cycles $(40 \mathrm{~ms})$ and the summed samples are weighted by a cosine function. HFR3 was a notch $50 \mathrm{~Hz}$ filter using the Fourier transform. HFR4 used a SavitzkyGolay FIR smoothing filter using the sgolayfilt function in Matlab with a polynomial order of 3 and a frame length of $15 \mathrm{~ms}$, HFR5 used rational transfer function for undesired noise removal [8]. The last two filters were Butterworth low pass filters with filter order 7, at two different cut-off frequencies, $30 \mathrm{~Hz}$ and $60 \mathrm{~Hz}$ respectively (HFR6 and HFR7 respectively) [9].

In the next step, four methods for baseline drift removal (BDR 1-4) were applied on the original signal and all processed signals. BDR1 used a wavelet bandpass filter with the decomposition performed over 20 wavelets and the filtering within $0.5-150 \mathrm{~Hz}$ band. In BDR2 method, a Savitzky-Golay FIR smoothing filter was used with a polynomial order of 3 and a frame length of $147 \mathrm{~ms}$. This estimation of the baseline wander was then subtracted from the original signals. BDR3 was a spline-based method using the predefined expected zero points of the measured signal for computation of the zero line by fitting the zero line with cubic function. The last method BDR4 was a Butterworth high pass filter of order 5 with a cut-off frequency of $0.5 \mathrm{~Hz}$. Using these signal processing procedures, a total of 32 variations of the original measured signals was obtained.
Finally, for all variations an averaged signal representing one heart beat was computed. The individual heart beats were specified in the time course of the selected tank signal by identification of R-peaks in ECG signal. The duration of the heart beat was defined manually by the proper time intervals around the R-peak. Then the heart beat signals were aligned according to the R-peak of each signal. The same time alignment was performed for all tank signals corresponding with one signal pre-processing variation. The averaged heart beat signal was then used for computation of the inverse problem of electrocardiology for estimation of the origin of ventricular pacing.

The origin of ventricular tachycardia was localized by the inverse solution to one dipole [10]. According to the basic presumption of the method, it is assumed that during the initial phase of activation only small area in the myocardium is activated, thus it can be represented by a single dipole. The body surface maps (BSPMs) used as input data for the inverse solution were computed for each time step from the $30 \mathrm{~ms}$ time interval from the beginning of the heart beat. The method then searched for an equivalent dipole (placed in one of the predefined positions in the ventricular muscle volume), which would best represent each input map. The quality of the obtained solution was evaluated by a relative residual error (RRE) between the input BSPM and the BSPM computed by the inversely estimated dipole. The position of the dipole giving the smallest RRE value from all predefined positions and for all considered time instants was put down as the localization of the origin of the observed electrical activation of the ventricles - the origin of ventricular pacing.

In the study the heart beats were artificially induced by intraventricular pacing in the known position of the stimulating electrode. Therefore, the localization error (LE) between the true stimulation point and the position of the origin of ventricular activation estimated by the inverse solution was evaluated for all possible combinations of the mentioned HFR and BDR signals preprocessing methods.

\section{Results}

The points defining the surface of the model of ventricles (epicardium) were used as the ventricular muscle predefined positions of the possible single dipole for the inverse solution. In Figure 2 the root-mean-squared (RMS) value of the original measured BSPM for each time instant is depicted. In Figure 3 up, the RMS of the original signal after application of HFR1 method and four BDR methods is shown. It is clearly visible that the baseline is constant for each BDR method, however it is considerably shifted from the zero line for BDR 1,2 and BDR4 methods. 



Figure 3. Up: RMS of the original signal after HFR1 processing and four baseline correction methods. Down: RMS of the averaged signal representing one heartbeat.

In Figure 3 down, the RMS of the averaged signal representing one heart beat after the mentioned signal preprocessing is displayed. The vertical line indicates the time instant assumed as the beginning of the ventricular activation. The position of a dipole best representing the input BSPMs was computed for all possible variations of the used BDR and HFR signal preprocessing methods. The LE values for all input data are summarized in the graph in Figure 4.

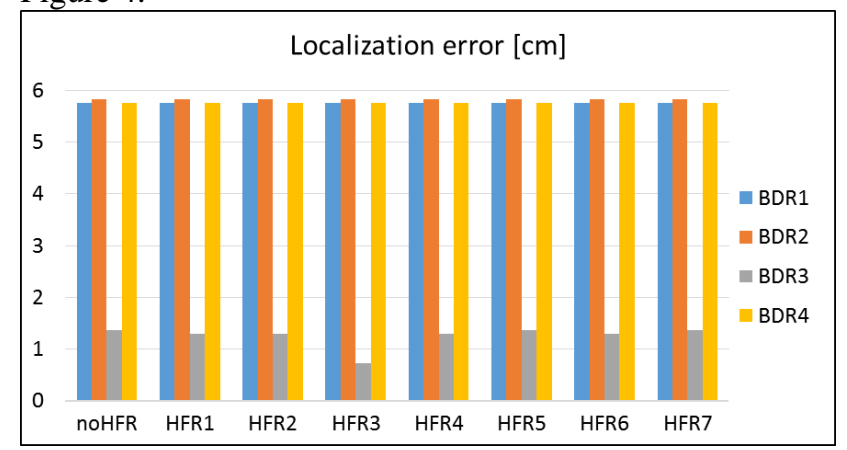

Figure 4. The LE between the known position of the electrode stimulating the VT and the inversely estimated origin of VT for 32 variations of signal preprocessing.

As it is apparent from the results, the large values of LE (more than $5 \mathrm{~cm}$ ) were obtained for the signals when the baseline did not correspond with the zero line (BDR1,2,4). For BDR3 the LE was significantly lower. Therefore, as the next step, the value of the averaged signal in the starting time instant of the studied time interval was regarded as a constant baseline shift and was subtracted from the rest of the signal. Such baseline correction aligned the baseline with the zero line. The RMS value of averaged signal from the Figure 3 after such correction is displayed in Figure 5.

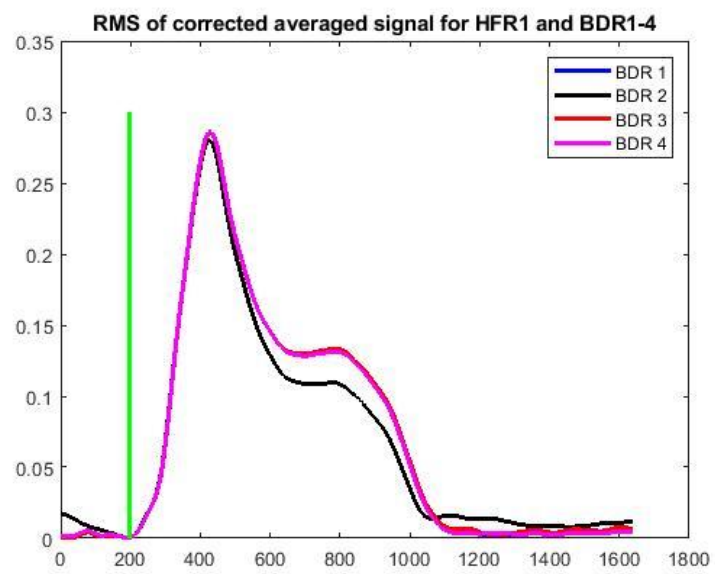

Figure 5. RMS of the averaged signal from Figure 3 after subtraction of a constant value from the beginning of the studied time interval.

Following these corrections, the LE of the inversely assessed origin of the heartbeats decreased considerably to values around $1 \mathrm{~cm}$ as it is demonstrated in Figure 6.

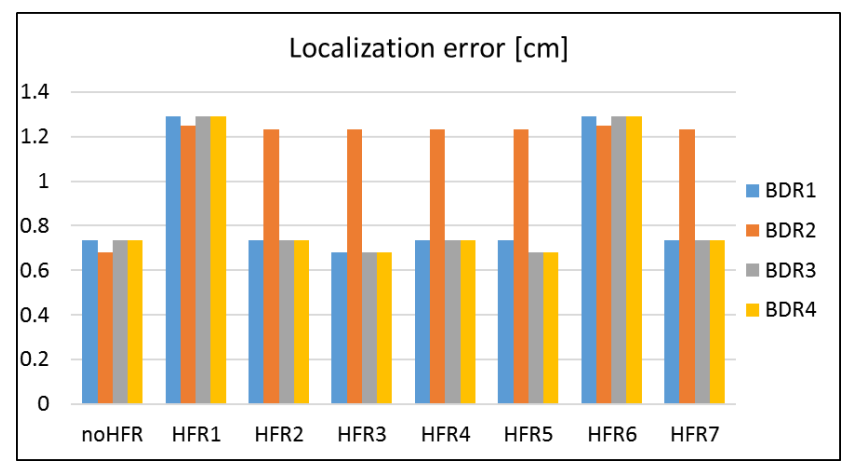

Figure 6. The LE between the known position of the electrode stimulating the VT and the inversely estimated origin of VT after baseline correction.

\section{Discussion and Conclusion}

The influence of signal preprocessing methods on the results of the inverse identification of the origin of ventricular pacing using a single dipole was studied on experimental signal. The signal was considerably disturbed by baseline wandering and strong $50 \mathrm{~Hz}$ power noise interference. From the results, it implies that the inverse method using a single dipole for localization of the origin of the heartbeat is very sensitive to the constant shift of the baseline and that it is almost not sensitive to the HFR methods. The results were the same even if no HFR 
method was used. One can object that the averaging of the signals can be considered as the additional HFR method. Indeed, a small random noise was eliminated by averaging. Curiously, the mentioned $50 \mathrm{~Hz}$ noise did not disappear after averaging as it is visible in Figure 7 where the RMS of the averaged signals for four BDR methods and no HFR are demonstrated.

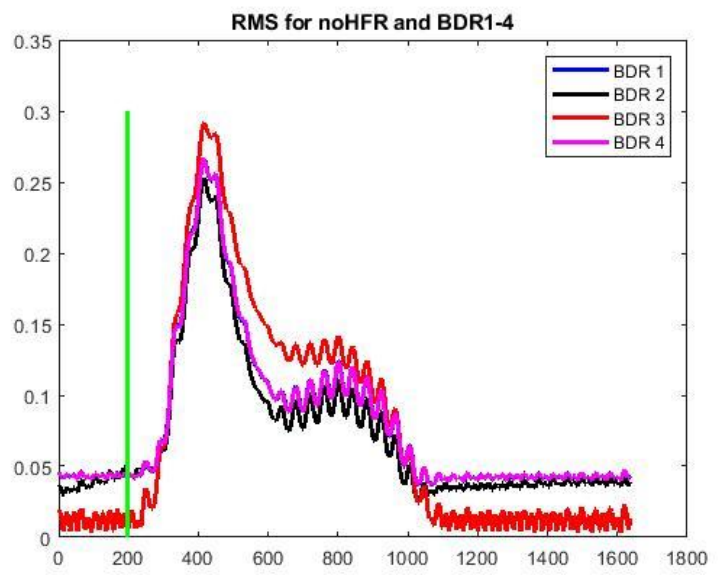

Figure 7. The remaining $50 \mathrm{~Hz}$ noise in averaged signal if no HFR method was performed.

The reason can be that in the studied experiment the ventricular activation was induced by an artificial pacing of the heart and the rhythm of pacing was multiple of the power frequency. For the averaging, the signals of all heartbeats were aligned according the R-peak what was always at the top of sinus of $50 \mathrm{~Hz}$ wave. The $50 \mathrm{~Hz}$ noise was not removed also by application of the HFR5 and HFR7 methods. On the other hand, as it can be seen from the results, the presence of this noise in the averaged signal did not worsen the LE of the inverse solution.

Because the inverse method using a single dipole needs to be applied to a very early time interval of ventricular activation, it is more sensitive to the proper selection of the zero-based baseline drift removal method than to removal of high frequency artefacts.

\section{Acknowledgements}

The study was performed within the ECGI Preprocessing Group, all authors contributed equally.

This work was supported by the research grant 2/0071/16 from the VEGA Grant Agency in Slovakia and by the grant APVV-14-0875 from the Slovak Research and Development Agency and in part by the French National Research Agency (ANR-10-IAHU04-LIRYC).

\section{References}

[1] Rudy Y. Noninvasive electrocardiographic imaging of arrhythmogenic substrates in humans. Circ Res 2013;112:863-74. doi:10.1161/CIRCRESAHA.112.279315.

[2] Cluitmans MJM, Peeters RL, Westra RL, Volders PG. Noninvasive reconstruction of cardiac electrical activity: update on current methods, applications and challenges. Netherlands Hear J 2015;23:301-11. doi:10.1007/s12471015-0690-9.

[3] Bear L, Cuculich PS, Bernus O, Efimov I, Dubois R. Introduction to noninvasive cardiac mapping. Card Electrophysiol Clin 2015;7. doi:10.1016/j.ccep.2014.11.015.

[4] Potyagaylo D, Segel M, Schulze WHW, Dössel O. Noninvasive localization of ectopic foci: A new optimization approach for simultaneous reconstruction of transmembrane voltages and epicardial potentials. Lect Notes Comput Sci (Including Subser Lect Notes Artif Intell Lect Notes Bioinformatics) 2013;7945 LNCS:166-73. doi:10.1007/978-3-642-38899-6_20.

[5] Van Dam PM, Tung R, Shivkumar K, Laks M. Quantitative localization of premature ventricular contractions using myocardial activation ECGI from the standard 12-lead electrocardiogram. J Electrocardiol 2013;46:574-9. doi:10.1016/j.jelectrocard.2013.08.005.

[6] Svehlikova J, Teplan M, Tysler M. Geometrical constraint of sources in noninvasive localization of premature ventricular contractions. J Electrocardiol 2018:\#pagerange\#. doi:10.1016/j.jelectrocard.2018.02.013.

[7] Stallmann FW, Pipberger H V. Automatic Recognition of Electrocardiographic Waves by Digital Computer. Circ Res 1961;IX:1138-43. doi:10.1161/01.RES.9.6.1138.

[8] Rodenhauser A, Good WW, Zenger B, Tate J, Aras K, Burton B, et al. PFEIFER: Preprocessing Framework for Electrograms Intermittently Fiducialized from Experimental Recordings. J Open Source Softw 2018;3:472. doi:10.21105/joss.00472.

[9] Rangayyan RM. Biomedical Signal Analysis. A Case-Study Approach. John Wiley \& Sons; 2002.

[10] Svehlikova J, Tysler M. Inverse Localization of Intraventricular Pacing Sites by Equivalent Dipole Source. Comput Cardiol (2010) 2017;44:1-4 doi:10.22489/CinC.2017.058-404.

Address for correspondence.

Jana Svehlikova

Institute of Measurement Science, Slovak Academy of Sciences Dubravska cesta 9, 84104 Bratislava, Slovakia

jana.svehlikova@savba.sk 